\title{
Endocervical Route of Administration
}

National Cancer Institute

\section{Source}

National Cancer Institute. Endocervical Route of Administration. NCI Thesaurus. Code C38205.

Administration of the drug within the canal of the cervix uteri. 